\title{
Wunderlich Syndrome in a patient with dengue infection - An entity to remember
}

\author{
Dayapala $\mathrm{A}^{1^{*}}$, Jayalath $\mathrm{P}^{2}$,Velu $\mathrm{M}^{3}$, Parthiepan $\mathrm{S}^{4}$.
}

\begin{abstract}
Dengue infection has become a serious public health concern. Dengue management protocols and the management of common complications arebeing updated often based on understanding the Pathophysiology of dengue infection. This understanding has definitely improved dengue management resulting in reduction in dengue deaths. At times, uncommon complications may be overlooked in busy and crowded clinical settings. Spontaneous localized retroperitoneal bleeding including bleeding into psoas muscle or Wunderlich Syndrome has been reported in few instances associated with dengue fever in this country and abroad. Thislife-threatening possibility must be born in mind when a clinician notices sudden deterioration of a dengue patient withoutsignificant thrombocytopenia, generalized bleeding manifestation or other evidence of extra vascular fluid loss. The management of this entity requires urgent restoration of the circulatory volume including urgent blood transfusion though, in general, clinicians are moreconcerned about fluid overload in the management of dengue patients. As awareness of this entity seems poor, further research and reporting of similar cases in medical literature will shed more light on this obscure entity and increase the awareness among clinicians with favorable results.
\end{abstract}

Key words:Retro-peritoneal bleeding, Wunderlichsyndrome, muscle bleeding, dengue fever

\section{Full paper}

\section{Introduction}

Spontaneous retroperitoneal bleeding or Wunderlich Syndrome is a rare but well recognized condition where non-traumatic bleeding occurring into the retroperitoneal tissues with signs and symptoms of varying severity. A number of causes have been identified including tumors in the kidneys and adrenal glands, visceral aneurysms, vasculitis, anticoagulation therapy and dengue infection. ${ }^{[1,2,3,4]}$ Here we report a case where sudden deterioration and death occurring in a patient with fever and dropping platelet counts without generalized bleeding or leaking manifestations. In the autopsy, retroperitoneal

${ }^{1}$ Judicial Medical Officer, Judicial medical Unit, ${ }^{2}$ Medical Unit, ${ }^{3}$ Haematology, ${ }^{4}$ Pathology Unit, Base Hospital, Avissawella,

*Corresponding author:Dayapala A, Tel: +94770701124, Email address: adayapala@yahoo.com

DOI: http://doi.org/10.4038/mljsl.v5i1.7348

https://orcid.org/0000-0002-7517-4908 bleeding was detected localized to the area adjacent to the left psoas muscle. ELISA on postmortem blood revealed Dengue IgM.

\section{Case history}

A 66-year-old, obese, female with diabetes, hypertension and dementia with depressive disorder was admitted to hospital on the second day of a febrile illness with brownish vomit. She had been on losartan, metformin,benzhexol, trifluoperazine and fluoxetine for about three years.

On admission, her blood pressure was 130/80 mmHg, the temperature 38.5 degree centigrade and capillary blood sugar $259 \mathrm{mg} / \mathrm{dl}$. Only paracetamol,ranitidine, omeprazole, metoclopramide,tranexamic acid and subcutaneous insulin according to capillary blood sugar were prescribed on admission.

On the second day as the blood pressure went up to 200/100mmHg, losartan was added. From second day onwards, drugs for mental illness were also added. Her condition remained stable up to the fourth day of 
illness. Her haemoglobin levels and platelets counts never dropped below 10.8g/dl and 105,000/cmm respectively during this period.

On the fourth day she suddenly developed difficulty in breathing and collapsed. Immediate cardio-

Blood counts after resuscitative effort showed a haemoglobin level of $4.2 \mathrm{~g} / \mathrm{dl}$ and pack cell volume of 22.3. Her platelets count was $65,000 / \mathrm{cmm}$. The blood picture was suggestive of a viral infection. Liver enzymes,AST and ALT levels were 7242IU/L and 8188IU/Lrespectively.She developed irreversible cardiac arrest within an hour after resuscitation.

At the autopsy, there was no evidence of trauma. The injection sites did not have excessive bleeding. Her conjunctivae and tongue were pale. The brain was pale and no intracranial haemorrhages were detected. The chest and pericardial cavities were free from effusion or bleeding.The heart was macroscopically unremarkable except some sub-endocardial bleeding in the left ventricle. Severe concentric atheroma was noted in coronaries. Oedematous lungs did not have evidence of pulmonary thrombo-embolism or bleeding.

Retro-peritoneal bleeding was found in the left psoas muscle extending into adjacent parts of the diaphragm, pelvic floor and the mesentery (Fig.1). The great vessels and branches had some atheroma. There was no intra-abdominal bleeding or peritoneal effusion.

The kidneys were granular, contacted and pale. The liver was dusky brown, soft and friable. The spleen

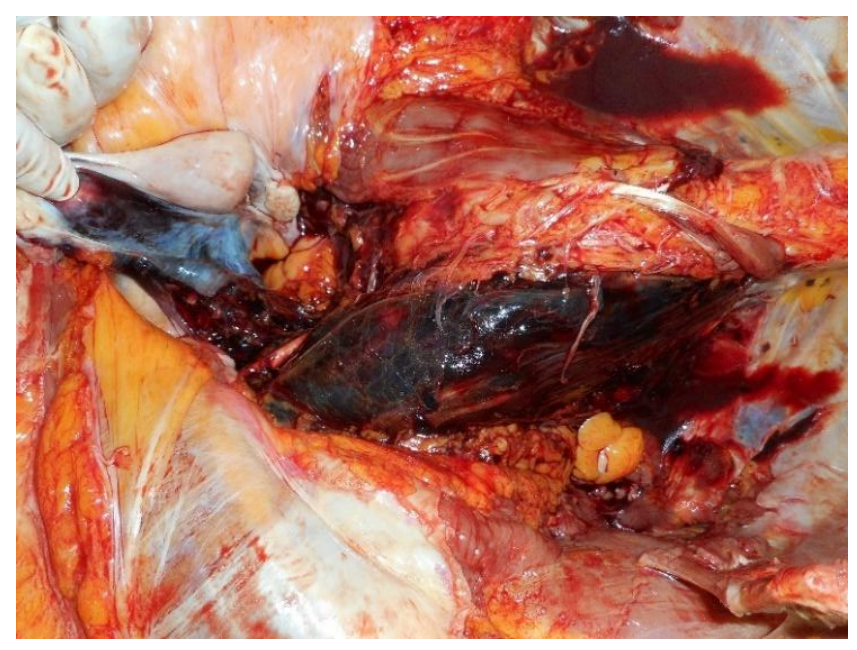

Figure 1: Retroperitoneal bleeding respiratory resuscitation was started with dopamine infusion. Her blood pressure picked up to 94/60mmHg with resuscitation.

was not enlarged but friable.The stomach had some dark brown fluid with mild mucosal erosion. No bleeding was noted in the bowels.

Histology showed bleeding into the muscle without inflammation in the blood vessels or surrounding tissues. Liver histology showed centrilobular necrosis and peri-portal inflammation, Kidneys had some glomerular sclerosis. Left ventricular myocardium showed patchy fibrosis. Right ventricular myocardium had significant fatty infiltration. Postmortem aerobic blood culture remained sterile. Dengue- IgM was detected in ELISA test on postmortem blood.

Pallor of organs, sub-endocardial hemorrhage and massive haematoma in relation to the left psoas muscle taken with the history of sudden collapse of the patient clearly indicate that the immediate cause of death is haemorrhagic shock. In the absence of other demonstrable cause, it is prudent to assume that the localized retroperitoneal haemorrhage is probably due to Wunderlich Syndrome caused by dengue infection is the underlying cause of death. Coronary atherosclerosis and microscopic evidence of myocardial fibrosis indicate preexisting myocardial ischemia as well. This condition could have contributed to her death. 


\section{Discussion}

The natural course of dengue infection has been well described. It ranges from asymptomatic infection, viral syndrome, dengue fever with or without bleeding to dengue haemorrhagic fever with or without shock. Unusual manifestations such as isolated organopathy involving the liver, kidneys, brain and the heart have also been described ${ }^{[5]}$ Dengue fever associated with localizedretroperitoneal or muscle bleeding has been reported in very few instances. ${ }^{[2,3,4,6]}$

In this casethe deceased had several co-morbidities which increase the mortality of dengue fever. ${ }^{5}$ She had not developed generalized bleeding manifestations or extra vascular fluid loss. We could exclude other recognized causes of retro-peritoneal bleeding such as trauma, tumors and vasculitis. Though some drugs, which she had been on, had thrombocytopenia as a side effect, they did not have anticoagulant properties. ${ }^{[7]}$ It was not possible to exclude small vascular malformations with available facilities.

The bleeding was retroperitoneal and localized as described in Wunderlich syndrome. ${ }^{[1]}$ It was in the left psoas muscle and extending to the adjacent structures retro-peritoneally. We could not find the origin of the bleeding.

As there were few documented cases of significant localized muscle bleeding associated with dengue fever, this possibility should be borne in mind when managing dengue cases. It will be useful in appropriately managing the patients who suddenly develop signs of shock without generalized bleeding or leaking manifestations.

\section{Conclusion}

Reporting of similar cases in medical literature and further research seems necessary to shed more light on this obscure entity and increase the awareness among clinicians.

\section{References}

1. Daliakopoulous SI. Spontaneous Retroperitoneal Hematoma; A Rare Devastating Clinical Entity. Journal of Surgical Technique and Case Reports. 2011 Jan. Jun.: 3(1) 8-9.

2. Koshy JM, John M, Rathore S, George UB. Spontaneous muscle hematomas in patients with Dengue hemorrhagic fever. CHRISMED Journal of Health Research 2014;1(3):201-2.

3. Bhat KJ, Shovkat R, Samoon HJ. Abdominal haematomas and dengue fever: two different cases of spontaneous psoas muscle haematoma and bilateral rectus sheath haematoma complicating dengue haemorrhagic fever. J Vector Borne Dis. 2015;52(4):339-41.

4. Ammer AM, Arachichi WK, Jayasingha PA. Psoas hematoma complicating dengue hemorrhagic fever: A case report. Galle Med J 2009;14:83-4.

5. Guidelines on Management of Dengue Fever \& Dengue Haemorrhagic Fever in Adults, Ministry of Health, Sri Lanka. National Guidelines Revised and Expanded Edition, November 2012.

6. Ganeshwaran Y, Senavirathne SM, Jayamaha R, De Silva AP, Balasuriya WK. Dengue fever associated with a Haematoma of rectus abdominis muscle. Ceylon Medical Journal 2001;46(3):105-6.

7. British National Formulary, 63, March 2012. London, Pharmaceutical Press. 2012. 